Check for updates

Cite this: RSC Adv., 2019, 9, 26547

\title{
Correction: Structural versatility of the quasi- aromatic Möbius type zinc(II)-pseudohalide complexes - experimental and theoretical investigations
}

\author{
Mariusz P. Mitoraj, ${ }^{\star a}$ Farhad Akbari Afkhami, ${ }^{b}$ Ghodrat Mahmoudi, ${ }^{* c}$ \\ Ali Akbar Khandar, ${ }^{\mathrm{b}}$ Atash V. Gurbanov, ${ }^{\text {de }}$ Fedor I. Zubkov, ${ }^{\text {f }}$ Rory Waterman, ${ }^{\mathrm{g}}$

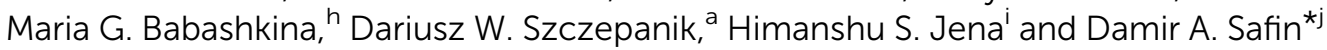 \\ Correction for 'Structural versatility of the quasi-aromatic Möbius type zinc(II)-pseudohalide complexes - \\ experimental and theoretical investigations' by Mariusz P. Mitoraj et al., RSC Adv., 2019, 9, 23764-23773.
}

DOI: $10.1039 /$ c9ra90062d

www.rsc.org/advances

The authors regret that the affiliations of Maria G. Babashkinah and Damir A. Safin were incorrectly shown in the original manuscript. The corrected list of affiliations is as shown herein.

The Royal Society of Chemistry apologises for these errors and any consequent inconvenience to authors and readers.

\footnotetext{
aDepartment of Theoretical Chemistry, Faculty of Chemistry, Jagiellonian University, Gronostajowa 2, 30-387 Cracow, Poland. E-mail: mitoraj@chemia.uj.edu.pl ${ }^{b}$ Department of Inorganic Chemistry, Faculty of Chemistry, University of Tabriz, 51666-16471, Tabriz, Iran

'Department of Chemistry, Faculty of Science, University of Maragheh, P.O. Box 55181-83111, Maragheh, Iran. E-mail: mahmoudi_ghodrat@yahoo.co.uk ${ }^{d}$ Department of Chemistry, Baku State University, Z. Xalilov Str. 23, AZ1148, Baku, Azerbaijan

${ }^{e}$ Centro de Quimica Estrutural, Instituto Superior Técnico, Universidade de Lisboa, Av. Rovisco Pais, 1049-001, Lisboa, Portugal

fOrganic Chemistry Department, Faculty of Science, Peoples' Friendship University of Russia (RUDN University), 6 Miklukho-Maklaya St., Moscow, 117198, Russian Federation ${ }^{8}$ Department of Chemistry, University of Vermont, 82 University Place, Burlington, VT 05405, USA

${ }^{h}$ Institute of Condensed Matter and Nanosciences, Université Catholique de Louvain, Place L. Pasteur 1, 1348 Louvain-la-Neuve, Belgium ${ }^{i}$ COMOC, Department of Chemistry, Ghent University, Krijgslaan 281-S3B, Ghent-9000, Belgium

${ }^{i}$ Innovation Center for Chemical and Pharmaceutical Technologies, Ural Federal University named after the First President of Russia B. N. Eltsin, Mira Str. 19, Ekaterinburg, 620002, Russian Federation. E-mail: damir.a.safin@gmail.com
} 